\title{
CFD Analysis of Temperature Distributions in a Slurry Bubble Column with Direct Contact Heat Transfer
}

\author{
Mohammed W. Abdulrahman \\ University of Ontario Institute of Technology \\ 2000 Simcoe St. N., Oshawa, Canada \\ mohammed.abdulrahman@uoit.ca
}

\begin{abstract}
Slurry bubble column reactors have different applications in the industry due to their advantages. In spite of the simple construction of the slurry bubble column reactors, their scale up analyses are complex due to the effect of various parameters on the hydrodynamic and heat transfer rates in these reactors. Direct-contact heat transfer in slurry bubble columns involves a complex phenomenon of bubble formation and gas motion through the slurry. In this paper, Computational Fluid Dynamics (CFD) simulations are used to investigate the temperature distributions for a direct contact heat transfer in a helium-water-alumina slurry bubble column, where helium gas is injected at $90^{\circ} \mathrm{C}$ through a slurry of water at $22^{\circ} \mathrm{C}$ and alumina solid particles. This paper studies the effects of superficial gas velocity, static liquid height, and solid particles concentration, on the average temperature of the slurry. In this study, it is assumed that the slurry inside the slurry bubble column is perfectly mixed, and the approaches used to model the slurry bubble column by CFD is $2 \mathrm{D}$ plane. From the CFD results, it is found that the average slurry temperature increases by increasing the superficial gas velocity and decreases by increasing the static liquid height and/or the solid concentration at any given superficial gas velocity, but the decrease with the solid concentration is negligible. The results of CFD simulations were compared with experimental data from the literature and show that the profiles of the slurry temperature calculated from CFD models, generally under-predicts the experimental data. The CFD model correctly predicts the experimental effects of static liquid height and solid concentration on average slurry temperature.
\end{abstract}

Keywords: slurry bubble column; direct contact heat transfer, temperature distribution, CFD, multiphase flow, solid concentration.

\section{Introduction}

Slurry bubble column reactors (SBCRs) are considered as multiphase reactors that have a wide variety of industrial applications which motivates doing more detailed studies with this type of reactors. Direct-contact heat transfer in a SBCR is defined as the heat transfer between the gas and the slurry phases without the presence of an intervening wall. In this way, heat transfer rate is increased because of the large interfacial area created by the dispersed gas phase. There are many parameters that affect the direct contact heat transfer rate in a SBCR, such as; gas flow rate, column dimensions, solid particles concentration, thermo physical properties of the gas and slurry phases, and the operating conditions of the column. Because of the complex interaction among the phases of the SBCR and the diverse parameters that affect the scale-up of the SBCR, the direct contact heat transfer in the SBCR have still not recognized very well.

In the literature, there are many studies related to the SBCRs, such as hydrodynamics, flow regimes, and heat transfer as well as design and scale up analyses. The studies of heat transfer in SBCRs have been reviewed by Nigar et al. [1] and Jhawar and Prakash [2]. It has been reported that the majority of the previous heat transfer studies in SBCRs were related to the steady-state condition heat transfer of two cases: wall-to-bed and immersed object-to-bed heat transfer [1]. Abdulrahman [3, 4, and 5] has performed experiments to study the hydrodynamic and direct contact heat transfer in a slurry bubble column with a high temperature helium gas $\left(90^{\circ} \mathrm{C}\right)$ that is injected through a slurry of liquid water and alumina solid particles at $22^{\circ} \mathrm{C}$. He has identified experimentally the transition velocity between the bubbly and churn-turbulent flow regimes [4]. Also, he has formulated empirical equations for the gas holdup [5] and the volumetric heat transfer coefficient [3] in terms of the Reynolds number, reactor dimensions and solid concentration, for both bubbly and churn-turbulent flow regimes.

Modeling by CFD simulations in multiphase flow has been studied by different researchers $[6,7,8,9,10]$. In the literature, there is no universal agreement that indicates that the CFD models are capable of predicting the experimental results of multiphase flow regimes [10]. For instance, Delnoij et al. [11, 12, 13] have used Eulerian-Lagrangian approach in 
a flat bubble column to model two-phase flow by using laminar flow model, as well as drag, lift, virtual-mass, and hydrodynamic-interaction forces [14]. Sokolichin and Eigenberger [15] have obtained same results by using finer grid size and neglecting the effects of virtual-mass and lift forces, as well as bubble-bubble interactions. Deen et al. [16] have found that using the virtual mass force will not influence the results. Krishna and Van Baten [17] have studied high pressure turbulent flow simulations and took into consideration the drag force only. They have found that there is high uncertainty when adding the effects of lift forces of small and large bubbles. Moreover, they have noted that there is no effect of the virtual mass force on the results of the simulations. Schwarz and Turner [18] have reported that, in a two phase flow, the agreement with the experimental results can be achieved without changing the values of the standard constants $\left(C_{1 \varepsilon}, C_{2 \varepsilon}, C_{3 \varepsilon}\right)$ of the $k-\varepsilon$ turbulence model. Sarah et al. [14] and Mahajan [19] have concluded that the 2-D Euler-Euler approach and the standard $k-\varepsilon$ turbulence model have been used in most of the literature, and most studies were limited to a churn-turbulent flow regime.

Abdulrahman [3] has explained in details the importance of using a SBCR with a high temperature helium gas to transfer the heat directly to the slurry of liquid water and solid alumina. In the literature, there is no reported study related to the CFD simulations of the temperature distributions in the SBCR with a direct contact heat transfer from a high temperature low density gas. Therefore, the lack of such study motivates the present work, which seeks to fill this gap by investigating numerically the direct contact heat transfer in a SBCR using helium gas at $90^{\circ} \mathrm{C}$ and alumina-water slurry at $22^{\circ} \mathrm{C}$. In this paper, the CFD simulations of the temperature distributions are performed for churn-turbulent flow regime only to study the effects of the bubble column design parameters, such as the reactor dimensions, superficial gas velocity, and solid concentration.

\section{CFD Analysis}

In bubble column reactors, the more common method used in the CFD of the multiphase flow is the Eulerian-Eulerian method with Eulerian sub-model, because of its accuracy [20]. Helium-water-alumina SBCR can be described by two phases: the slurry continuous (primary) phase and the dispersed (secondary) gas phase. The flow in the helium-water-alumina bubble column reactor is incompressible and Newtonian. In 2D Cartesian coordinates, the conservation equations are written as (conservation equations for slurry phase have the same form of that for gas phase, so they are not repeated);

Continuity equation [21]:

$$
\frac{\partial v_{x, g}}{\partial x}+\frac{\partial v_{y, g}}{\partial y}=0 \quad(\text { gas phase })
$$

Momentum equations [22]:

$$
\begin{array}{ll}
\mathrm{x}-\text { direction: } & \rho_{g} \alpha_{g}\left(\frac{\partial v_{x}}{\partial t}+v_{x} \frac{\partial v_{x}}{\partial x}+v_{y} \frac{\partial v_{x}}{\partial y}\right) \\
& =-\alpha_{g} \frac{\partial P}{\partial x}+\alpha_{g} \frac{\mu_{g, e f f}}{3} \frac{\partial(\nabla \cdot \boldsymbol{V})}{\partial x}+\mu_{g, e f f} \alpha_{g}\left[\frac{\partial^{2} v_{x}}{\partial x^{2}}+\frac{\partial^{2} v_{x}}{\partial y^{2}}\right]+\rho_{g} \alpha_{g} g_{x}+M_{i, g, x} \\
\mathrm{y}-\text { direction: } & \rho_{g} \alpha_{g}\left(\frac{\partial v_{y}}{\partial t}+v_{x} \frac{\partial v_{y}}{\partial x}+v_{y} \frac{\partial v_{y}}{\partial y}\right) \\
& =-\alpha_{g} \frac{\partial P}{\partial y}+\alpha_{g} \frac{\mu_{g, e f f}}{3} \frac{\partial(\nabla \cdot \boldsymbol{V})}{\partial y}+\mu_{g, e f f} \alpha_{g}\left[\frac{\partial^{2} v_{y}}{\partial x^{2}}+\frac{\partial^{2} v_{y}}{\partial y^{2}}\right]+\rho_{g} \alpha_{g} g_{y}+M_{i, g, y}
\end{array}
$$

Where $P$ is the pressure that is shared by all phases, $\mu_{e f f}$ is the effective dynamic viscosity, $g$ is the gravitational acceleration and $M_{i}$ is the total interfacial force acting between the phases. The subscript $g$ represents the gas phase.

Energy equation [23]:

$$
\alpha_{g} \rho_{g} C\left(v_{x, g} \frac{\partial T_{g}}{\partial x}+v_{y, g} \frac{\partial T_{g}}{\partial y}\right)=\overline{\bar{\tau}}_{g}: \nabla \boldsymbol{V}_{g}+k_{g}\left(\frac{\partial^{2} T_{g}}{\partial x^{2}}+\frac{\partial^{2} T_{g}}{\partial y^{2}}\right)+S_{g}+Q_{g, s l}
$$


where $h$ is the specific enthalpy of each phase, $\overline{\bar{\tau}}$ : $\nabla \boldsymbol{V}$ is the viscous stress tensor contracted with the velocity gradient, $S$ is a source term that includes sources of enthalpy and $Q_{g, s l}$ is the intensity of heat exchange between the gas and slurry phases [23].

In the modeling of turbulence, the standard $k-\varepsilon$ model is widely used in the CFD simulations of hydrodynamics and heat transfer of SBCRs, because of its reasonable accuracy and simplicity. The derivation of the standard $k-\varepsilon$ model was based on the assumption that the flow is fully turbulent, and the effects of molecular viscosity are negligible [20]. Since the CFD simulations of this paper are for churn turbulent flow only, the most suitable turbulence model that can be used is the standard $k-\varepsilon$ model. The standard $k-\varepsilon$ model comprises three turbulence sub models: the mixture turbulence model, the dispersed turbulence model, and a per-phase turbulence model [20]. In this paper, the dispersed turbulence model is used because of its less computational efforts required than the per-phase turbulence model and because of the large density ratio between helium gas and water that restricts the use of the mixture model [20]. The wall function that is used in this paper is the standard wall function, where all the meshes should be created corresponding to a target $\mathrm{y}^{+}$between 30 and 300 (i.e. within the log-law layer of the boundary layer).

\section{Domain Description and ANSYS FLUENT V.13 Setup}

The numerical model of the helium-water-alumina SBCR was based on using 2D-CFD simulations which were run using the ANSYS FLUENT V.13 software for a column with an overall diameter of $21.6 \mathrm{~cm}$ and three different heights of static liquid $(45,55$, and $65 \mathrm{~cm})$. Quadratic structured meshes were used for the areas of the SBCRs and fine meshes were used near the wall with $\mathrm{y}^{+}$values ranges between 62 and 75 . The size of the mesh was selected so that to get the grid independence of the average slurry temperature $\left(\bar{T}_{s l}\right)$. After investigating the grids for different $H$ and $U_{g s}$, it has been found that the most unfavourable situation is for $H=65 \mathrm{~cm}$ and $U_{g s}=0.15 \mathrm{~m} / \mathrm{s}$. In this paper, the grid convergence index (GCI) method is used to study the effect of the mesh size on the average slurry temperature, by using three different mesh sizes. Table 1 shows the grid independence study that was used to select the best grid distribution of the bubble column problem, where the solid concentration $\left(C_{s}\right)$ is zero. From the GCI method, it was found that the extrapolated solution of $\bar{T}_{s l}$ (theoretical solution for an infinitely fine mesh) is $302.48 \mathrm{~K}$ and the numerical uncertainty in the grid solution for $\bar{T}_{s l}$ is $0.22 \%$.

Table 1: Grid independence test for a helium-water BC $\left(H=65 \mathrm{~cm}, U_{g s}=0.15 \mathrm{~m} / \mathrm{s}\right)$.

\begin{tabular}{|c|c|c|c|}
\hline Total Cells & 5,047 & 10,128 & 20,235 \\
\hline $\bar{T}_{s l}(K)$ & 299.4 & 301.8 & 301.95 \\
\hline
\end{tabular}

Fig. 1a shows the convergence of $\bar{T}_{s l}$ for different mesh sizes towards the extrapolated solution as given by the GCI method. From Fig. 1, it can be seen that the best grid is the finest one, where the number of cells is 20,235 cells. Therefore, the finest mesh is used in the CFD simulations of this paper. Fig. 1b shows the details of the mesh used for the SBCR with $21.6 \mathrm{~cm}$ inside diameter and $65 \mathrm{~cm}$ static liquid height. The quantities of interest that were monitored during the CFD simulation were the inlet gas temperature, outlet gas temperature and slurry average temperature. The convergence criteria of the simulation was to ensure that the quantities of interest were reached to a steady state simulation and the residual RMS error values were less than $10^{-4}$.

In this paper, PC-SIMPLE algorithm is used for the Eulerian multiphase flow. The gradients are computed according to the Least Squares Cell-Based method, where the accuracy of this method is higher than that of the node-based gradient for regular structured meshes (as it is used in this work). Also, it is less expensive to compute the least-squares gradient than the node-based gradient. For details about least-squares gradient method refer to [20].

\section{Boundary Conditions}

The boundary conditions of the SBCR can be represented by inlet, outlet and wall boundary conditions. The inlet volume fraction of the gas is equal to 1 and the inlet velocity of the gas is considered uniform and equal to the volumetric flow rate of the gas divided by the total cross-sectional area of the sparger's orifices.

According to Akhtar et al. [24], the pressure boundary condition can be applied at the outlet of the column because it will produce better convergence. In all simulations, the outlet pressure is equal to atmospheric pressure. The no-slip boundary 
conditions are applied at the walls of the SBCR. Symmetry conditions were not used in the simulations to be able to obtain better behaviors of hydrodynamics and heat transfer. Because of the estimation difficulty of the liquid turbulence at the inlet and outlet boundary conditions of the liquid phase, iterations were used to specify the turbulent kinetic energy and dissipation rate.

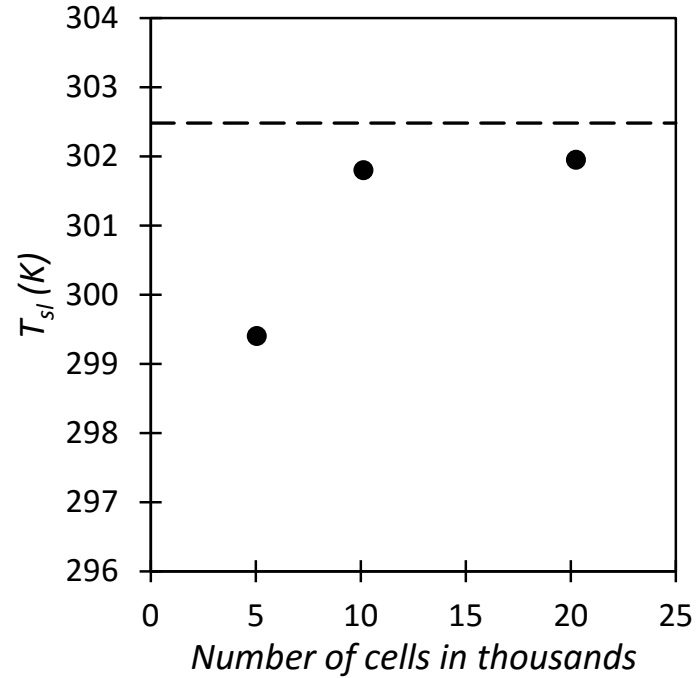

(a)

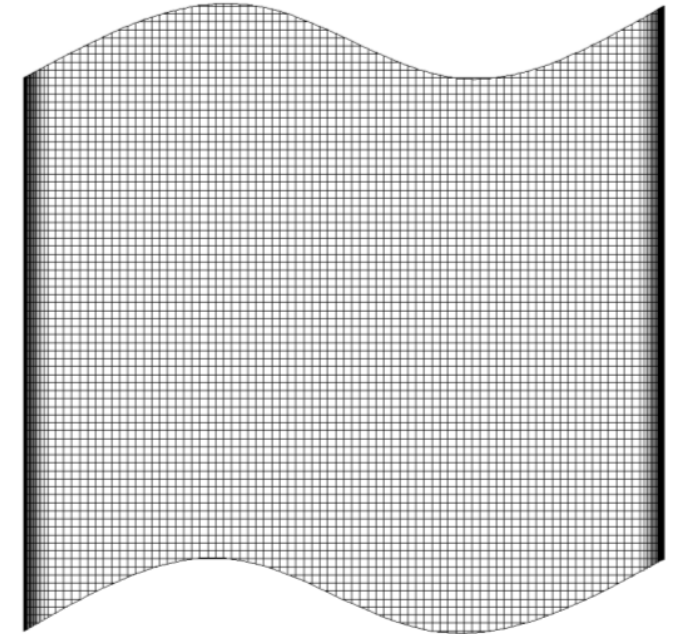

(b)

Fig. 1: a) Average slurry temperature for different mesh sizes $\left(C_{s}=0 \%, H=65 \mathrm{~cm}, U_{g s}=0.15 \mathrm{~m} / \mathrm{s}\right)$, b) Details of mesh used for the 2D-plane of SBCR.

\section{Results}

Some assumptions are made in the CFD simulations. First, the slurry is assumed to be perfectly mixed. This is true if Stokes number $(S t k)$ of solid particles is small $(S t k \ll 1)$. Stokes number is calculated from [25];

$$
S t k=\frac{\rho_{p} d_{p}^{2} U_{l}}{18 \mu_{l} D_{R}}
$$

where $\rho_{s}$ and $d_{p}$ are the density $\left(\mathrm{Kg} / \mathrm{m}^{3}\right)$ and diameter $(\mathrm{m})$ of the solid particles respectively, $\mu_{l}$ is the dynamic viscosity of the surrounding liquid, $D_{R}$ is the reactor diameter, and $U_{l}$ is the liquid velocity which is taken here as the same value of the largest superficial gas velocity $\left(U_{g s}=0.15 \mathrm{~m} / \mathrm{s}\right)$. In this way, it can be ensured that the Stokes number will not exceed the calculated value from Eq. (5). For the slurry of the liquid water and activated alumina solid particles that was used in the experimental work of Abdulrahman [3], the Stokes number was calculated to be in the range of 0.1-0.6 for the range of $U_{g s}$ between 0.03 and $0.15 \mathrm{~m} / \mathrm{s}$. From the above values, it can be seen that the Stokes number of Activated alumina with water is not much smaller than one. In this case, the accuracy error will by higher, where it has been found that, if Stk $\ll 0.1$, accuracy errors are below 1\% [26]. The approaches that is used in this paper is the 2D plane. These approaches can be used when the static liquid height is less than 4 column diameter, because there will be no bubble plume oscillation in the column and it can be modeled as 2D plane [25]. Since CFD simulations don't achieve highly smoothed curves, therefore, all the curves of the CFD results are fitted by using a second order polynomial with $\mathrm{R}^{2}$ higher than 0.99 .

\subsection{Effect of Superficial Gas Velocity $\left(U_{g s}\right)$ and Static Liquid Height $(H)$ on $\bar{T}_{s l}$}

To examine the effect of $U_{g s}$ and $H$ on $\bar{T}_{s l}$, CFD simulations were performed for a range of $U_{g s}$ from 0.03 to $0.15 \mathrm{~m} / \mathrm{s}$ and different $H(45,55$, and $65 \mathrm{~cm})$. Fig. 2 shows the variation of $\bar{T}_{s l}$ with $U_{g s}$ and $H$ in the water-helium BCR with $C_{s}=0$. In this figure, it can be seen that $\bar{T}_{s l}$ increases by increasing $U_{g s}$ and/or decreasing $H$. The increase of $\bar{T}_{s l}$ with $U_{g s}$ is attributed to the increase in the gas flow rate that leads to higher relative velocities between gas bubbles and liquid, and hence higher 
heat transfer rates. The results show that $\left(\bar{T}_{s l}\right)$ increases by about $0.48 \%$, when increasing $U_{g s}$ velocity from 0.1 to $0.15 \mathrm{~m} / \mathrm{s}$ for $H=65 \mathrm{~cm}$. The decrease of $\bar{T}_{s l}$ with increasing $H$ is attributed mainly to the increase of slurry volume and hydrostatic pressure when increasing $H$ which will cause a decrease in the mixing rate of the slurry. From the results of Fig. 2, it can be seen that, for $C_{s}=0 \%$, the rate of decrease of $\bar{T}_{s l}$ ranges from 0.54 to $1.17 \%$ (for $U_{g s}=0.03$ to $0.15 \mathrm{~m} / \mathrm{s}$ ), when $H$ increases from 45 to $65 \mathrm{~cm}$.

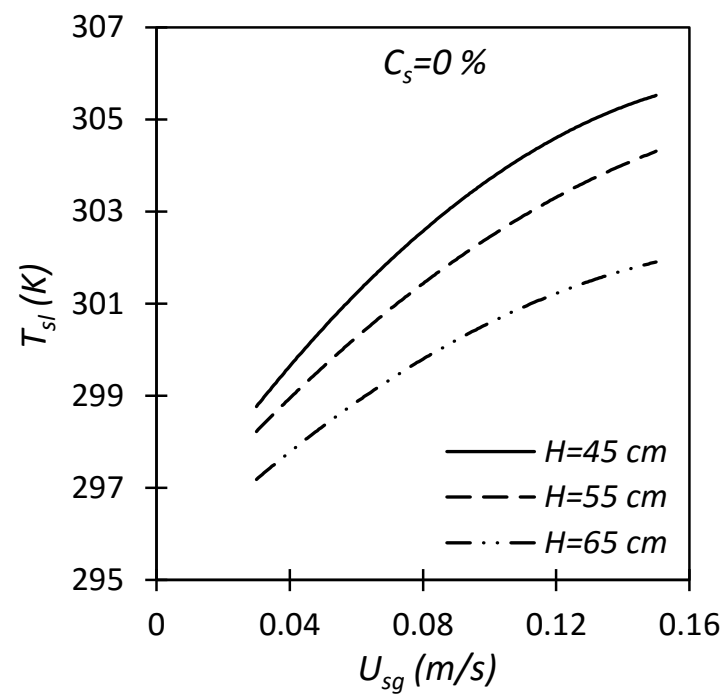

(a)

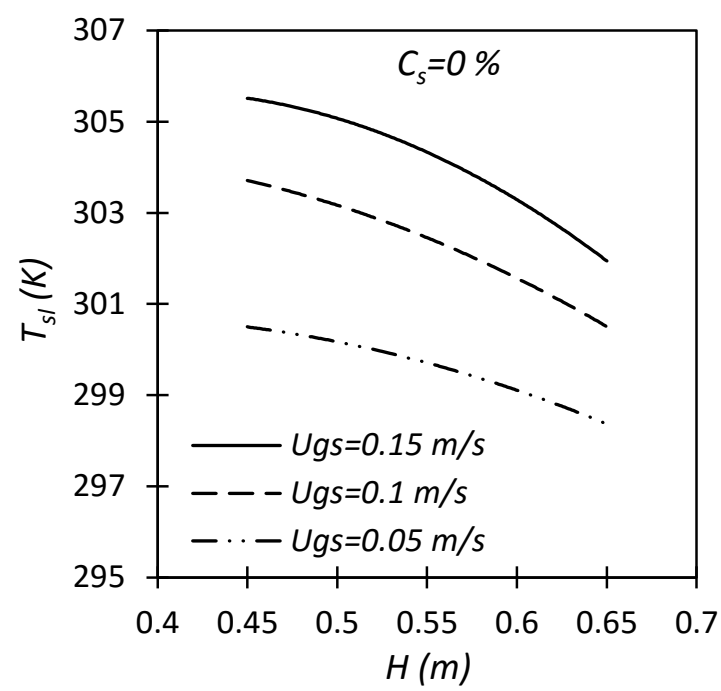

(b)

Fig. 2: Effect of $H$ and $U_{g s}$ on $\bar{T}_{s l}$ for $C_{s}=0 \%$.

Fig. 3 shows the contours of the gas temperature $\left(T_{g}\right)$ for a helium-water BCR with $C_{s}=0 \%$ and $H=65 \mathrm{~cm}$ for different $U_{g s}$. In this figure, it can be seen that the major reduction in $T_{g}$ occurs near the bottom of the reactor. For the remaining height of the reactor, the decrease in $T_{g}$ is insignificant and the temperature can be considered as constant. This means that gas transfers most of the heat to the liquid near the bottom of the reactor, which reflects the fact that direct contact heat transfer between the gas and liquid is very efficient.

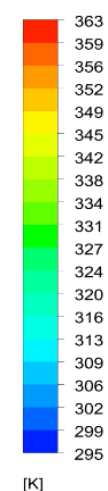

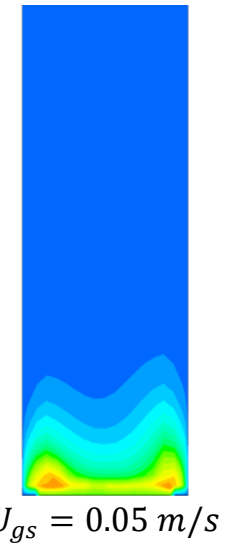
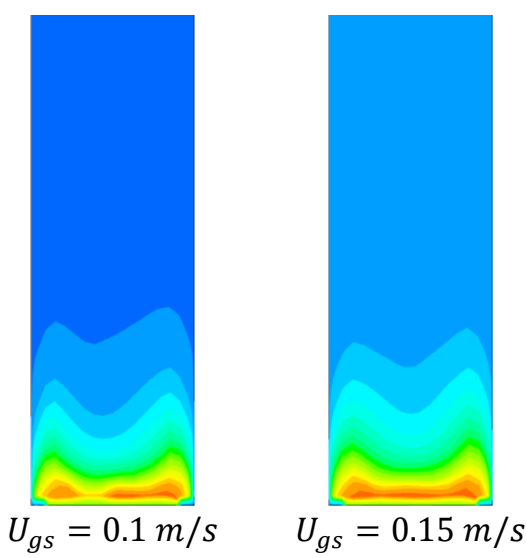

Fig. 3: Axial gas temperature contours for different $U_{g s}$ of helium-water BC with $C_{s}=0$ and $H=65 \mathrm{~cm}$.

\subsection{Effect of Solid Concentration $\left(C_{s}\right)$ on $\bar{T}_{s l}$}

Figure 4 shows the effect of $C_{s}$ on $\bar{T}_{s l}$ for $H=45 \mathrm{~cm}$. From this figure, it can be seen that $\bar{T}_{s l}$ decreases insignificantly by increasing $C_{s}$. For instance, in the case of $H=45 \mathrm{~cm}$ and $C_{s}=0 \%, \bar{T}_{s l}$ is equal to $305.5 \mathrm{~K}$ at $U_{g s}=0.15 \mathrm{~m} / \mathrm{s}$, while at $C_{s}=5 \%$, it is equal to $305.1 \mathrm{~K}$ and at $C_{s}=10 \%$ is equal to $304.8 \mathrm{~K}$ at the same $U_{g s}$. The decrease in $\bar{T}_{s l}$ by increasing $C_{s}$ 
can be attributed to the increase of large bubbles and then decrease of gas holdup when increasing $C_{s}$. This will lead to the decrease of the heat transfer rate between the gas and slurry.

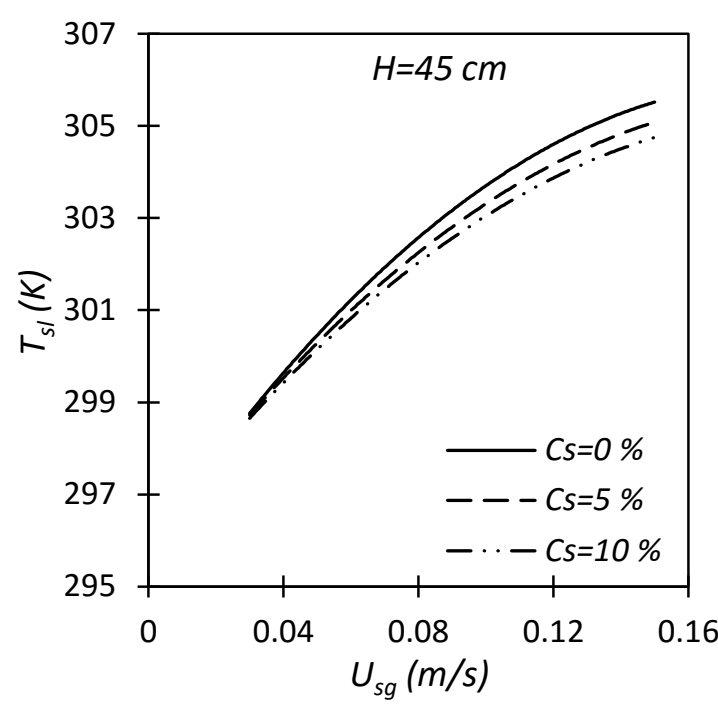

(a)

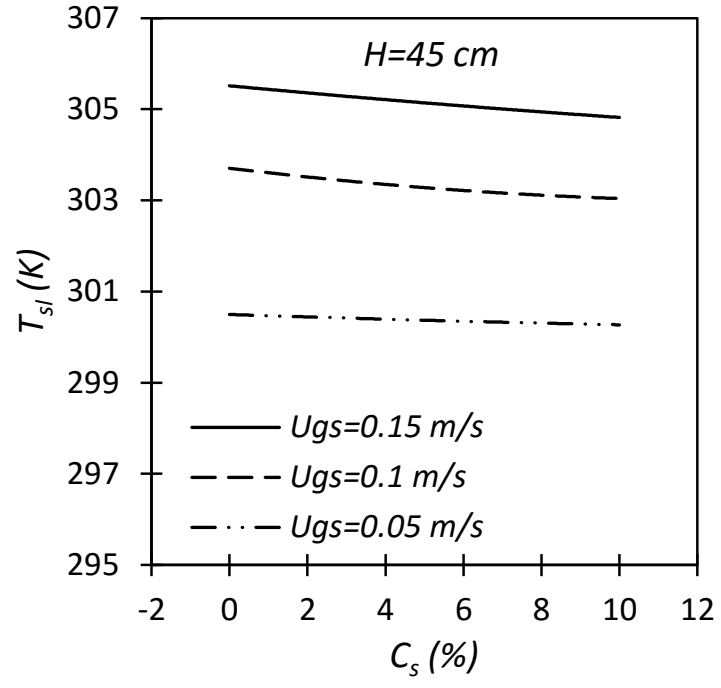

(b)

Fig. 4: Effect of $C_{s}$ and $U_{g s}$ on $\bar{T}_{s l}$ for $H=45 \mathrm{~cm}$.

\subsection{Comparison of Numerical $\bar{T}_{s l}$ with the Experimental Data}

To validate the results of the CFD simulations of the helium-water-alumina SBCR, a comparison is performed with the experimental data obtained from [3]. Figs. $5 \& 6$ show the comparisons of $\bar{T}_{s l}$ variations with $U_{g s}, H$ and $C_{s}$. From these figures, it can be seen that all profiles of $\bar{T}_{s l}$ calculated from CFD models generally under-predict the experimental data, with a maximum relative error of less than $0.4 \%$. The reduction in the numerical values of the $\bar{T}_{s l}$ is due to the use of a 2D-plane mesh which produces lower gas flow rates and then lower heat transfer rate when compared with the 3D mesh. Also, CFD models neglect the impact of the sparger height used in the experiments. Therefore, they over-estimates the static liquid height compared with the experimental one.

Figs. $5 \& 6$ also show that the experimental rates of decrease of $\bar{T}_{s l}$ with $H$ and $C_{s}$ are correctly predicted by CFD models. From Fig. 5, it can be seen that, at a specific $C_{s}$, the rate of change of $\bar{T}_{s l}$ versus $U_{g s}$ is different for each $H$, where the rates of decrease of $\bar{T}_{s l}$ with $H$ increase by increasing $U_{g s}$. For example, at $C_{s}=0 \%, \bar{T}_{s l}$ decreases by $0.54 \%$, when $H$ increases from $45 \mathrm{~cm}$ to $65 \mathrm{~cm}$ at $U_{g s}=0.03 \mathrm{~m} / \mathrm{s}$, while it decreases by $1.17 \%$ at $U_{g s}=0.15 \mathrm{~m} / \mathrm{s}$. From Fig. 6 , it can be seen that, at a specific $H$, the rate of change of $\bar{T}_{s l}$ versus $U_{g s}$ is different for each $C_{s}$, where the rates of decrease of $\bar{T}_{s l}$ with $C_{s}$ increase by increasing $U_{g s}$. For example, at $H=45 \mathrm{~cm}, \bar{T}_{s l}$ decrease by $0.06 \%$, when $C_{s}$ increases from $0 \%$ to $10 \%$ at $U_{g s}=0.03 \mathrm{~m} / \mathrm{s}$, while it decreases by $0.23 \%$ at $U_{g s}=0.15 \mathrm{~m} / \mathrm{s}$.

\section{Conclusions}

In this paper, CFD simulations were performed on a helium-water-alumina SBCR to examine the effects of superficial gas velocities $\left(U_{g s}\right)$, static liquid heights $(H)$ and solid concentrations $\left(C_{s}\right)$ on the average slurry temperature $\left(\bar{T}_{s l}\right)$ of a direct contact heat transfer. In this paper, it was assumed that the slurry inside SBCR is perfectly mixed, and the approaches used to model SBCR by CFD were 2D plane. From the results of this paper, it was found that the average slurry temperature $\left(\bar{T}_{s l}\right)$ increases by increasing $U_{g s}$ and/or decreasing $H$. Also, it was found that $\bar{T}_{s l}$ increases insignificantly by decreasing $C_{s}$. The results of this paper were compared with a previous experimental data and it was shown that the CFD profiles of $\bar{T}_{s l}$, generally under-predicted the experimental data. However, the CFD models showed good agreements with the previous experimental data and correctly predicted the experimental effects of $H$ and $C_{s}$ on $\bar{T}_{s l}$. 


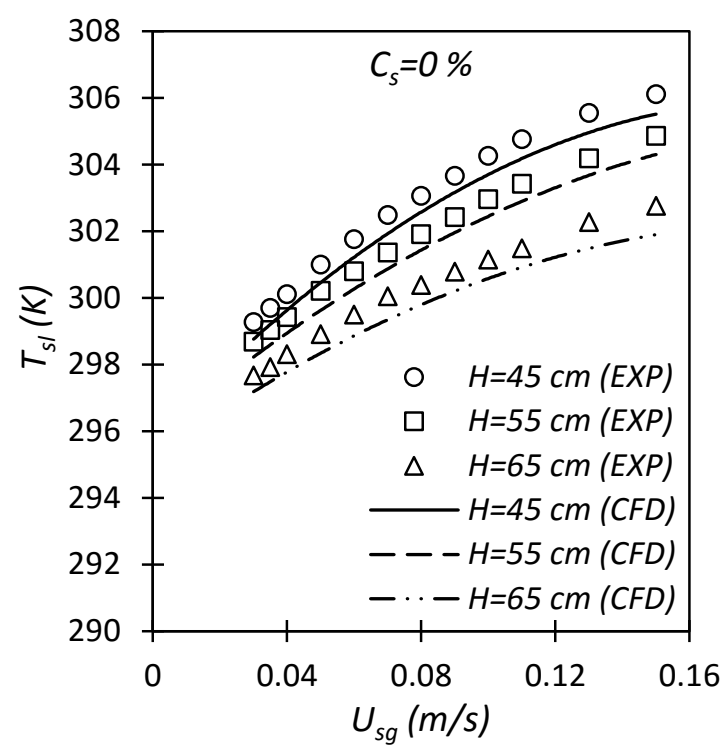

(a)

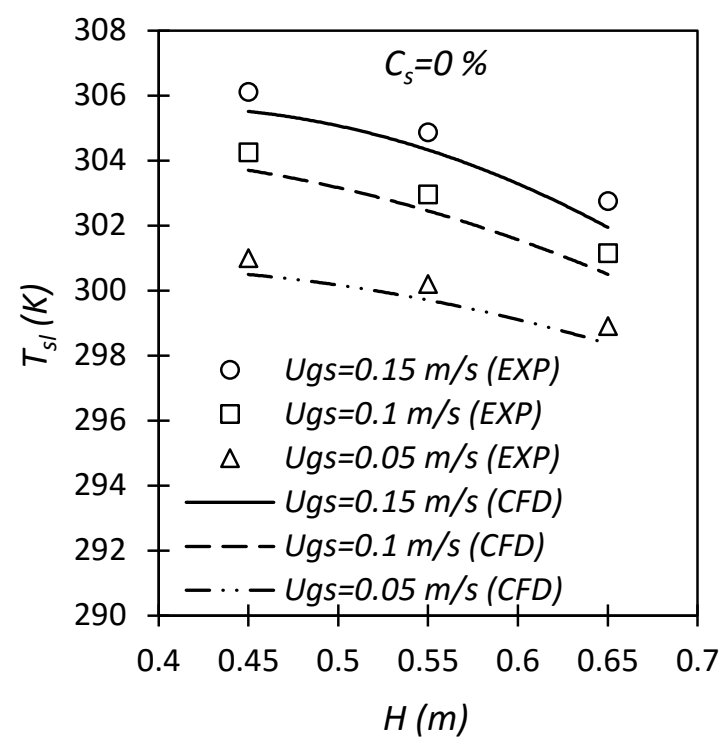

(b)

Fig. 5: Comparison between numerical and experimental $\bar{T}_{s l}$ versus $U_{g s}$ and $H$.

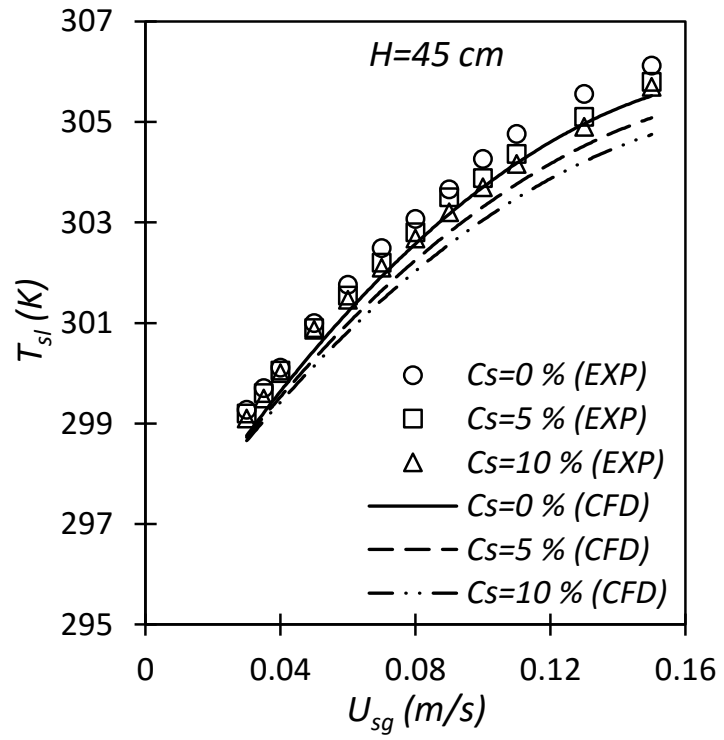

(a)

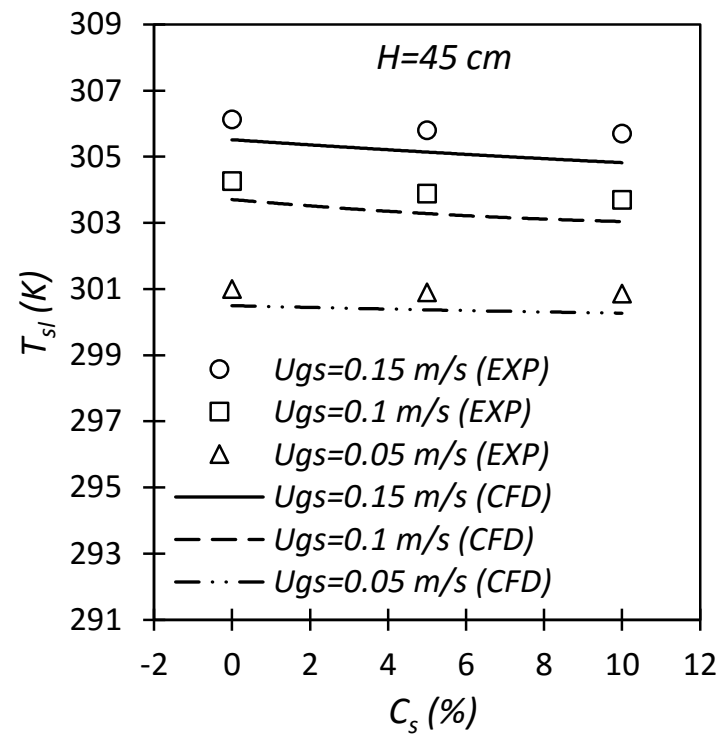

(b)

Fig. 6: Comparison between numerical and experimental $\bar{T}_{s l}$ versus $U_{g s}$ and $C_{s}$.

\section{References}

[1] K. Nigar, B. Fahir, and O. U. Kutlu, "Review: Bubble column reactors," Process Biochem., vol. 40, pp. 2263-2283, 2005.

[2] A. K. Jhawar and A. Prakash, "Heat Transfer in a Slurry Bubble Column Reactor: A Critical Overview," Ind. Eng. Chem. Res., vol. 51, pp. 1464-1473, 2012.

[3] M. W. Abdulrahman, "Experimental studies of direct contact heat transfer in a slurry bubble column at high gas temperature of a helium-water-alumina system," Appl. Therm. Eng., vol. 91, pp. 515-524, 2015.

[4] M. W. Abdulrahman, "Experimental studies of the transition velocity in a slurry bubble column at high gas temperature of a helium-water-alumina system," Exp. Therm. Fluid Sci., vol. 74, pp. 404-410, 2016 a.

[5] M. W. Abdulrahman, "Experimental studies of gas holdup in a slurry bubble column at high gas temperature of a helium-water-alumina system," Chem. Eng. Res. Des., vol. 109, pp. 486-494, 2016 b. 
[6] C. Chen and L. S. Fan, "Discrete simulation of gas-liquid bubble columns and gas-liquid-solid fluidized beds," AIChE $J$, vol. 50, pp. 288-301, 2004.

[7] J. B. Joshi, "Computational flow modelling and design of bubble column reactors," Chem. Eng. Sci., vol. 56, pp. 58935933, 2001.

[8] J. B. Joshi, V. S. Vitankar, A. A. Kulkarni, M. T. Dhotre, and K. Ekambara, "Coherent flow structures in bubble column reactors," Chem. Eng. Sci., vol. 57, pp. 3157-3183, 2002.

[9] S. La1'n, D. Bröder, M. Sommerfeld, and M. Göz, "Modelling hydrodynamics and turbulence in a bubble column using the Euler-Lagrange procedure," Int. J. Multiphase Flow, vol. 28, pp. 1381-1407, 2002.

[10] A. Sokolichin, G. Eigenberger, and A. Lapin, "Simulation of buoyancy driven bubbly flow: Established simplifications and open questions," AIChE J, vol. 50, pp. 24-45, 2004.

[11] E. Delnoij, J. A. M. Kuipers, and W. P. M. van Swaaij, "Computational fluid dynamics applied to gas-liquid contactors," Chem. Eng. Sci., vol. 52, pp. 3623-3638, 1997a.

[12] E. Delnoij, J. A. M. Kuipers, and W. P. M. van Swaaij, "Dynamic simulation of gas-liquid two-phase flow: Effect of column aspect ratio on the flow structure," Chem. Eng. Sci., vol. 52, pp. 3759-3772, 1997b.

[13] E. Delnoij, F. A. Lammers, J. A. M. Kuipers, and W. P. M. van Swaaij, "Dynamic simulation of dispersed gas-liquid two-phase flow using a discrete bubble model," Chem. Eng. Sci., vol. 52, pp. 1429-1458, 1997c.

[14] M. M. Sarah, S. V. Vivek, and O. F. Rodney, "CFD predictions for flow-regime transitions in bubble columns," AIChE $J$, vol. 51, no. 7, pp. 1897-1923, 2005.

[15] A. Sokolichin and G. Eigenberger, "Applicability of the standard k $-\varepsilon$ turbulence model to the dynamic simulation of bubble columns: Part I. Detailed numerical simulations," Chem. Eng. Sci., vol. 54, p. 2273-2284, 1999.

[16] N. G. Deen, T. Solberg, and B. H. Hjertager, "Large eddy simulation of gas-liquid flow in a square cross-sectioned bubble column," Chem. Eng. Sci., vol. 56, pp. 6341-6349, 2001.

[17] R. Krishna, J. M. van Baten, M. I. Urseanu, and J. Ellenberger, "Design and scale-up of the bubble column slurry reactor for Fischer-Tropsch synthesis," Chem. Eng. Sci., vol. 56, p. 537, 2001.

[18] M. Schwarz and W. Turner, "Applicability of the standard k $-\varepsilon$ turbulence model to gas-stirred baths," Appl. Math. Modell., vol. 12, no. 3, pp. 273-279, 1988.

[19] V. Mahajan, "CFD analysis of hydrodynamics and mass transfer of a gas-liquid bubble column," Bachelor's project, Rourkela, Orissa, India, 2010.

[20] ANSYS, ANSYS FLUENT Theory Guide, Release 14.5. ANSYS, Inc., 2012.

[21] A. Bejan, Convection heat transfer ( $3^{\text {rd }}$ ed.). John Wiley \& Sons, Inc., 2004.

[22] G. K. Batchelor, An introduction to fluid dynamics. Cambridge, England: Cambridge University Press, 1967.

[23] A. Bejan, Advanced engineering thermodynamics ( $2^{\text {nd }}$ ed.), New York: Wiley, 1997.

[24] M. A. Akhtar, M. O. Tadé, and V. K. Pareek, "Two-fluid Eulerian simulation of bubble column reactors with distributers," J. Chem. Eng. Jpn., vol. 39, no. 8, pp. 831-841, 2006.

[25] P. Chen, J. Sanyal, and M. P. Dudukovik, "Numerical simulation of bubble columns flows: effect of different breakup and coalescence closures," Chem. Eng. Sci., vol. 60, pp. 1085-1101, 2005.

[26] C. Tropea, A. L. Yarin, and J. F. Foss, Springer Handbook of Experimental Fluid Mechanics. Springer, 2007. 